\title{
ARTICLE \\ Influence of gut microbiome on mucosal immune activation and SHIV viral transmission in naive macaques
}

\author{
Yongjun Sui ${ }^{1}$, Amiran Dzutsev ${ }^{2}$, David Venzon ${ }^{3}$, Blake Frey ${ }^{1}$, Vishal Thovarai ${ }^{2}$, Giorgio Trinchieri ${ }^{2}$ and Jay A. Berzofsky ${ }^{1}$
}

It is unknown whether the gut microbiome affects HIV transmission. In our recent SHIV vaccine study, we found that the naive rhesus macaques from two different sources had significantly different rates of infection against repeated low-dose intrarectal challenge with SHIV $\mathrm{SF}_{162 \mathrm{P} 4}$ virus. Exploring causes, we found that the more susceptible group of seven macaques had significantly more activated $\mathrm{CD} 4^{+} \mathrm{CCR} 5{ }^{+} \mathrm{Ki}^{+} 7^{+} \mathrm{T}$ cells in the rectal mucosa than the more resistant group of 11 macaques from a different source. The prevalence of pre-challenge activated rectal CD4 T cells in the naive macaques correlated inversely with the number of challenges required to infect. Because the two naive groups came from different sources, we hypothesized that their microbiomes may differ and might explain the activation difference. Indeed, after sequencing $16 \mathrm{~s}$ rRNA, we found differences between the two naive groups that correlated with immune activation status. Distinct gut microbiota induced different levels of immune activation ex vivo. Significantly lower ratios of Bacteroides to Prevotella, and significantly lower levels of Firmicutes were found in the susceptible cohort, which were also inversely correlated with high levels of immune activation in the rectal mucosa. Thus, hostmicrobiome interactions might influence HIV/SIV mucosal transmission through effects on mucosal immune activation.

Mucosal Immunology (2018) 11:1219-1229; https://doi.org/10.1038/s41385-018-0029-0

\section{INTRODUCTION}

The most common routes of HIV transmission are through the genital and rectal mucosa. The gastrointestinal (GI) tract, as the primary viral replication site and viral reservoir for HIV-1, plays an important role in the viral control and eradication. Recent studies demonstrated that the $\mathrm{Gl}$ tract harbors over 100 trillion bacteria, which form a unique, and functionally stable community, termed the gut microbiome. ${ }^{1}$ The gut microbiome not only facilitates the breakdown of food for energy metabolism, but also contributes to the development and functionality of the immune system, responses to infections, and even behavioral abnormalities..$^{2-5}$ The cross-talk between the gut microbiome and intestinal immune system is critical for the maintaining of mucosal homeostasis. $^{2}$

Though the mechanisms by which commensal bacteria modulate the immune system have not been fully elucidated, recent advances in gut microbiome research revealed that a range of bacteria and their derivatives can influence immune functions through intestinal epithelial cells, mononuclear phagocytes, innate lymphoid cells, and T and B lymphocytes. ${ }^{6}$ A large number of T lymphocytes is present in intestine to perform regulatory and effector functions. ${ }^{7}$ Commensal bacteria and their metabolites in the Gl tract can promote different cell subset induction. ${ }^{8-10}$ Among them, short-chain fatty acid (SCFA) metabolites derived from commensal bacteria can induce intestine $\mathrm{FoxP}^{+}$Treg cells directly. ${ }^{11-13}$ Capsular polysaccharide A (PSA) of Gram-negative anaerobic bacteria Bactericides fragilis have been shown to be able to induce Treg cells through TLR2 as well. ${ }^{14,15}$ Besides T cells, some bacteria can regulate the CC chemokine responsiveness including CCR5 in vitro in monocytes. ${ }^{16}$ Thus, distinct members of the gut microbiome or their metabolites can regulate the prevalence of certain immune cell types, and the immune activation markers on these cells.

It is well documented that the gut mucosal homeostasis is interrupted during HIV/SIV infections. HIV/SIV infections affect the gut microbiome, even in the elite controllers, ${ }^{17}$ and the altered gut microbiome in turn leads to general immune activation via bacterial translocation. ${ }^{18-23}$ However, it is not known whether the reciprocal is true, that the gut microbiome affects susceptibility to HIV/SIV infection. As immune activation also affects the HIV/SIV susceptibility, this raises the interesting question whether naive animals with different gut microbiomes will have different risk of HIV/SIV infection via the modulation of local mucosal immune activation status. In this study, we sought to investigate the associations of gut microbiome with rectal immune activation markers and HIV/SIV rectal transmission using two cohorts of naive rhesus macaques from either Morgan Island or Alpha Genesis Inc. Coming from different sources, the two naive cohorts displayed different gut microbial compositions, which correlated with the viral target cells and with the immune activation markers/status in the colorectal mucosa. Ex vivo treatment of PBMCs with heat-killed fecal bacterial samples from these two cohorts led to different expression levels of immune activation markers on $\mathrm{CD}^{+}{ }^{+} \mathrm{T}$ cells. When we challenged these macaques simultaneously with repeated low-dose of $\mathrm{SHIV}_{\mathrm{SF} 162 \mathrm{P} 4}$ virus via the intrarectal route, we observed that they had significantly different rates of infection ( $47 \%$ vs $21 \%$ per exposure, ${ }^{*} P=0.04$ ). In addition, the viral target cells and the immune activation status in the colorectal mucosa inversely correlated with the number of exposures needed for the animals to get infected. Through these associations and the ex vivo experiment, our data suggested that the different gut microbiota

\footnotetext{
${ }^{1}$ Vaccine Branch, National Cancer Institute, National Institutes of Health, Bethesda, MD 20892, USA; ${ }^{2}$ Cancer and Inflammation Program, National Cancer Institute, National Institutes of Health, Bethesda, MD 20892, USA and ${ }^{3}$ Biostatistics and Data Management Section, National Cancer Institute, National Institutes of Health, Bethesda, MD 20892, USA Correspondence: Yongjun Sui (suiy@mail.nih.gov) or Jay A. Berzofsky (berzofsj@mail.nih.gov)
}

Received: 8 November 2017 Revised: 23 March 2018 Accepted: 2 April 2018

Published online: 1 June 2018 


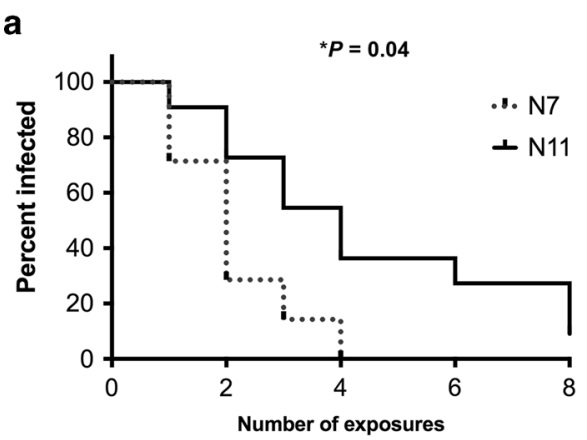

b
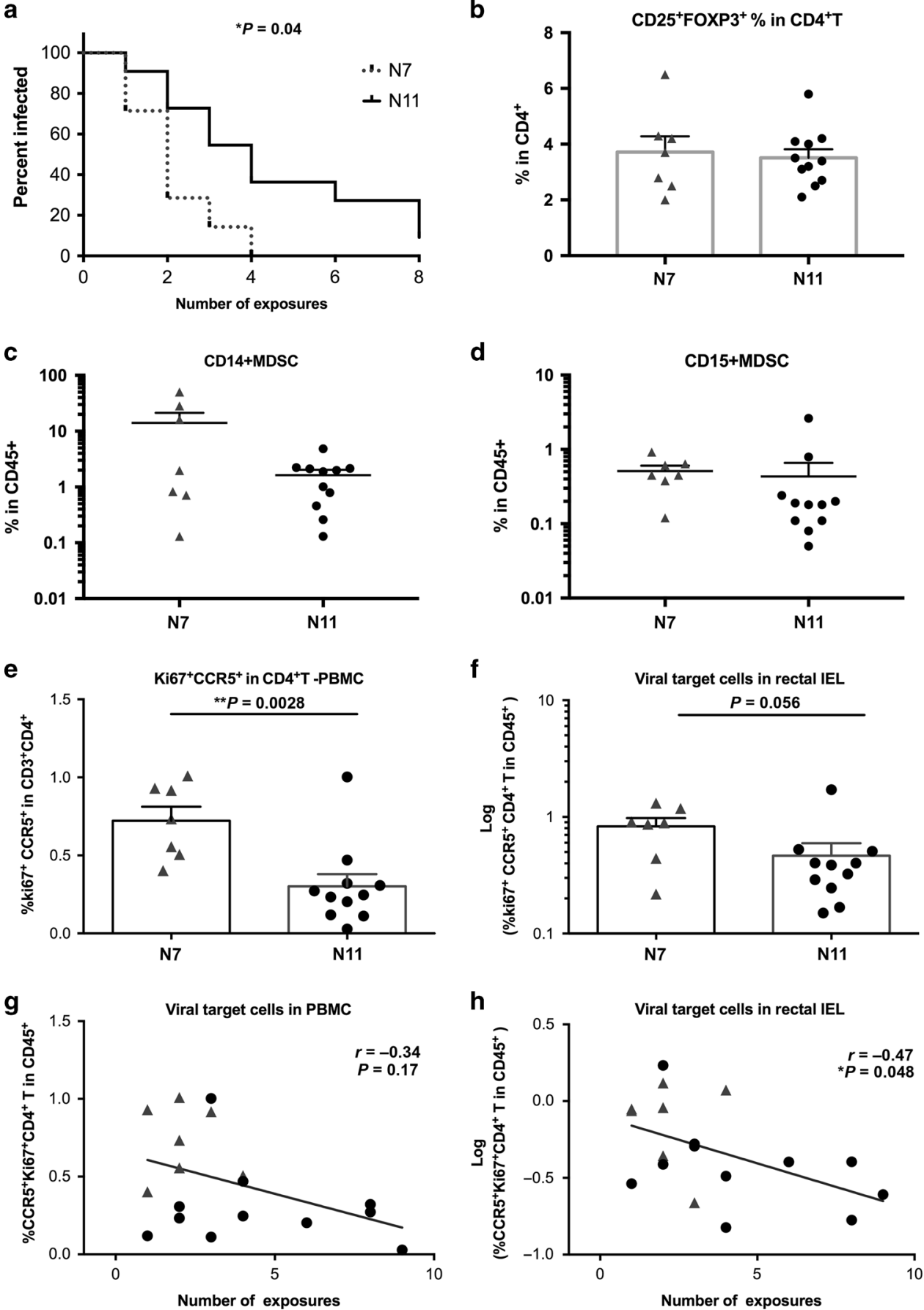

Fig. 1 Viral target cells in the rectal IEL correlated with SHIV acquisition. a Viral acquisition curves from two naive cohorts (N7 and N11) were

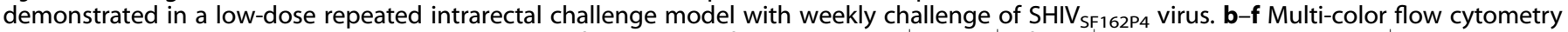
techniques were used to measure and compare the frequencies of rectal LP CD25 $5^{+} \mathrm{FOXP3} 3^{+}$of CD4 ${ }^{+} \mathrm{T}$ cells (b), rectal LP CD14 ${ }^{+} \mathrm{MDSCs}(\mathbf{c})$ and CD15 ${ }^{+}$MDSCs (d) cells; as well as the viral target cells (Ki67 ${ }^{+}$CCR5 ${ }^{+}$in CD4 ${ }^{+}$T cells) in PBMC (e) and rectal IEL (f) in N7 and N11 cohorts. MannWhitney tests were used for comparisons. g, h Correlations between viral target cells in PBMC (g) and rectal IEL (h) and number of viral exposures for the animals to get infected were performed using Spearman's tests. Mean \pm SEM are shown. Triangles denote the N7 group, and filled circles denote the N11 group

might affect the infection risk of HIV-1 via the rectal mucosal immune activation status, including the availability of viral target cells. Though commensal bacteria have been shown to be involved in modulating immune responses in the $\mathrm{Gl}$ tract, this is the first demonstration of the association of the gut microbiome with susceptibility to HIV/SIV viral infection in a macaque model. This could have profound implications to develop strategies to prevent HIV-1 transmission.

\section{RESULTS}

Two cohorts of naive macaques, which demonstrated different SHIV viral infection rates, had different viral target cells in the rectal mucosa

Two cohorts of naive Indian rhesus macaques were obtained from two different sources: the N7 group of seven animals were wildcaptured from Morgan Island, while the N11 group of 11 animals were inbred from Alpha Genesis Inc (Supplementary Table 1). 
Before administration of SHIV $\mathrm{SF} 162 \mathrm{P} 4_{4}$ viral challenges, the N7 and the N11 group of macaques were cohoused in the same room for 5 months. The repeated low-dose simian/human immunodeficiency virus (SHIV) challenge macaque model has been widely used to assess the HIV susceptibility. ${ }^{24,25}$ In this study, we utilized this model to test the viral susceptibility of these two cohorts of naive macaques. We gave the macaques low-dose SHIV SF162P4 $_{1}$ virus intrarectally every week for 8 weeks. After 8 SHIV challenges, 17 out of 18 animals were infected, and the infection rate for all the animals was $27 \%$. However, we observed that these two cohorts demonstrated significantly different infection rates. N7 had an infection rate of $47 \%$ per exposure, while $\mathrm{N} 11$ had only a $21 \%$ rate. Even with the small number of animals in each cohort, they showed a significant difference in viral acquisition $(P=0.04$, Fig. 1a).

To explore the possible cause of this difference, we examined different cell subsets and the immune activation markers that are associated with the immune regulation in the colorectal tissues. Since $\mathrm{CD}^{+}$regulatory $\mathrm{T}$ cells $\left(\mathrm{T}_{\text {regs }}\right)$ are present at higher frequencies in the colon lamina propria (LP) than in other organs, ${ }^{26}$ and have important implications in inflammatory bowel disease, we first measured the frequencies of Tregs in the rectal mucosa. We found that the frequency of $\mathrm{CD}_{2} 5^{+} \mathrm{FOXP}^{+}$cells among $\mathrm{CD}^{+}{ }^{+}$cells was about $4 \%$, and no significant difference in Treg cell frequencies in the colon LP between the two cohorts were observed (Fig. 1b, Supplementary Figure 1).

We then tested myeloid-derived suppressor cells (MDSC), which not only are important immune regulators to suppress the immune activation, but also have been identified as viral target cells in the SIV-infected macaques. ${ }^{27}$ We measured two major subsets of MDSCs in the colorectal LP, and found that neither $\mathrm{CD}_{14}^{+}$MDSCs nor $\mathrm{CD}^{+} 5^{+}$MDSCs showed any significant difference between the two cohorts (Fig. 1c, d, Supplementary Figure 2). MDSC did not correlate with viral acquisition either.
We next measured the $\mathrm{CD}^{+} \mathrm{T}$ viral target cell $\left(\mathrm{CD} 4^{+} \mathrm{CCR} 5^{+} \mathrm{Ki} 67^{+} \mathrm{T}\right)$ frequencies in the blood and gut mucosal tissues based on our and other's studies. ${ }^{28-30}$ We have shown previously that the viral target cells in the rectal mucosa determined the viral loads and viral eclipse time once the animals were infected, while Carnathan et al. found that the activated $\mathrm{CD}^{+}{ }^{+} \mathrm{CCR}^{+}{ }^{+} \mathrm{T}$ cells in the rectum predicted increased SIV acquisition in SIVGag/Tat-vaccinated rhesus macaques. ${ }^{28-30}$ Indeed, we found that the original more susceptible N7 group had more activated $\mathrm{CD} 4^{+} \mathrm{CCR} 5^{+} \mathrm{Ki} 67^{+} \mathrm{T}$ cells (viral target cells) in PBMC $(P=0.003)$, and in the rectal intraepithelial lymphocytes (IEL) $(P=0.056)$ than those in the more resistant group of $\mathrm{N} 11$ (Fig. 1e-f, Supplementary Figure 3). Though the frequency of viral target cells in the rectal mucosa of the N7 group only showed a trend $(p=0.056)$ of higher levels than that of the N11 group, it significantly correlated inversely with the number of intrarectal exposures needed for viral infection (Fig. 1h). Viral target cells in the PBMC showed a trend, but did not significantly correlate with viral acquisition (Fig. 1g). This supported the conclusion that the viral target cells in the rectal mucosa played an important role in determining viral transmission.

The mucosal immune activation status was significantly different in the two cohorts of macaques

Besides viral target cells, we further explored whether there were other immune activation markers that could be used to more precisely define the immune activation status of the rectal mucosa. We have measured five immune activation markers (Ki67, CD69, CD38, CCR5, and HLA-DR), which have been associated with HIV-1 infections, in three cell subsets $\left(C D 4^{+} \mathrm{T}\right.$, $\mathrm{CD}^{+} \mathrm{T}$, and $\mathrm{CD} 14^{+}$monocytes). To generalize the idea, we analyzed the data using an unsupervised principal component analysis (PCA) with Qlucore Omics Explorer. ${ }^{31}$ With the first three PCA components explaining $67 \%$ of the total variance, the

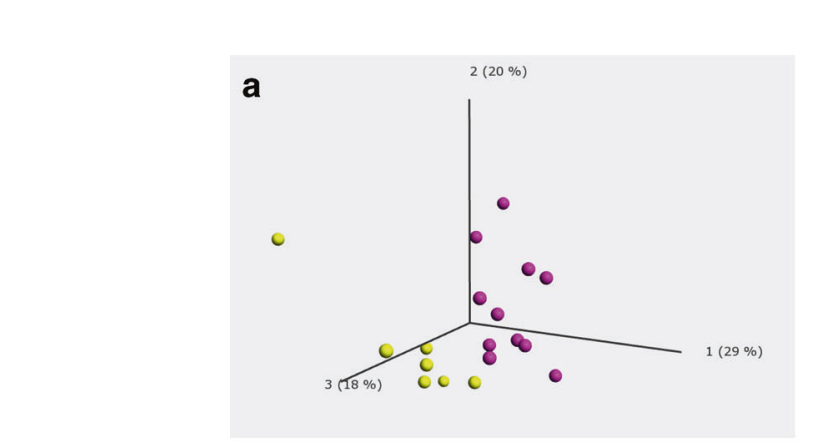

C PCA-1 for Rectal immune activation

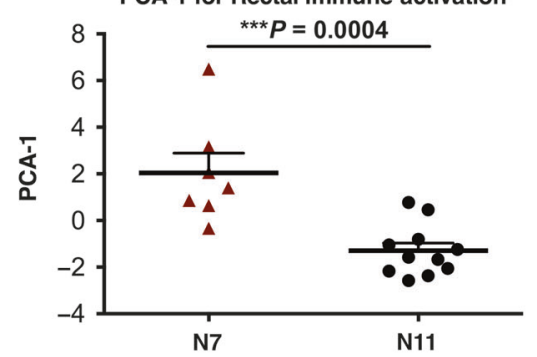

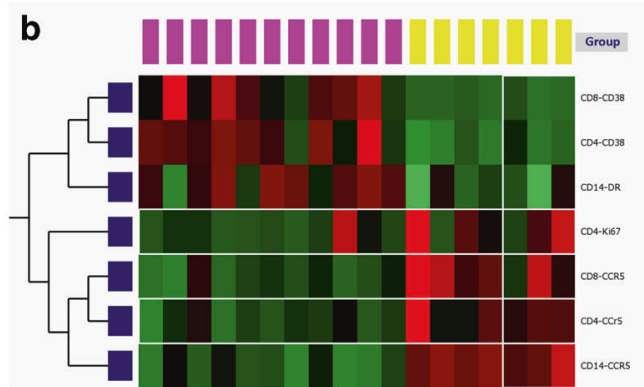

d

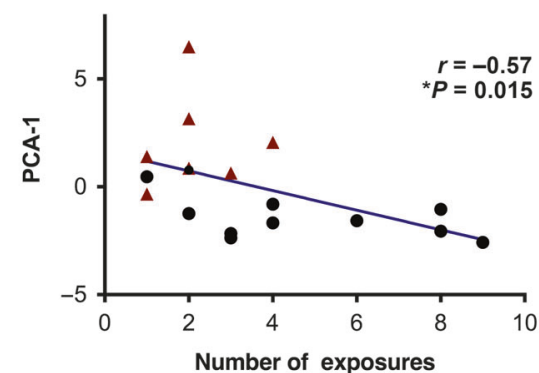

Fig. 2 The immune activation status was significantly different in the two naive groups. a The frequencies of Ki67, CD69, CD38, HLA-DR, and CCR5 in $\mathrm{CD}^{+}, \mathrm{CD}^{+} \mathrm{T}$ cells, and $\mathrm{CD} 14^{+}$monocytes, as well as the percentages of the three cell subsets, were used to create a principal component analysis (PCA) plot. Magenta dots denote the N7 group, and yellow dots denote the N11 group. b The signature immune activation markers of the two cohorts (N7 and N11) were shown. c Immune activation PCA-1 was exported and compared between the N7 and N11 cohorts using a Mann-Whitney test. Mean \pm SEM are shown. $\mathbf{d}$ Correlation between immune activation PCA-1 and numbers of viral exposures required for the animals to get infected were performed using Spearman's test. The Qlucore Omics Explorer (Qlucore) was used to perform the PCA 
a

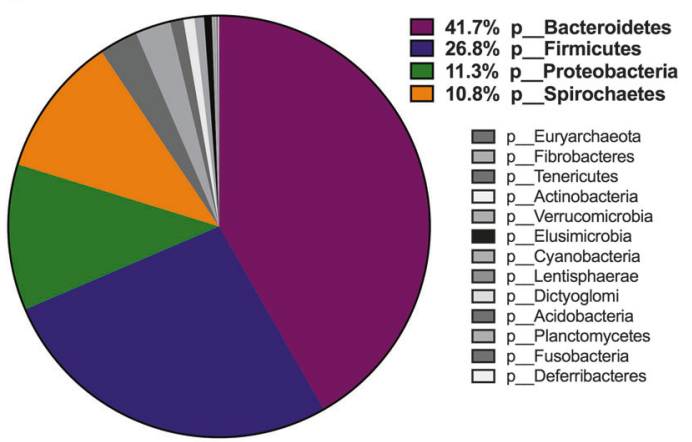

b

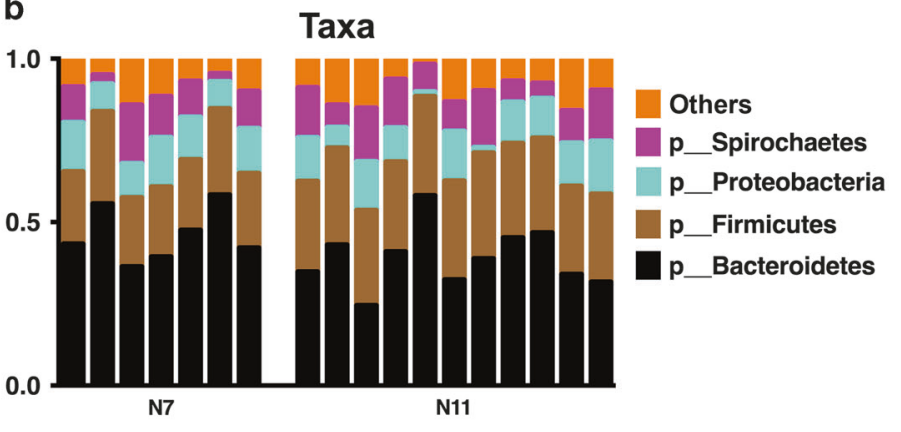

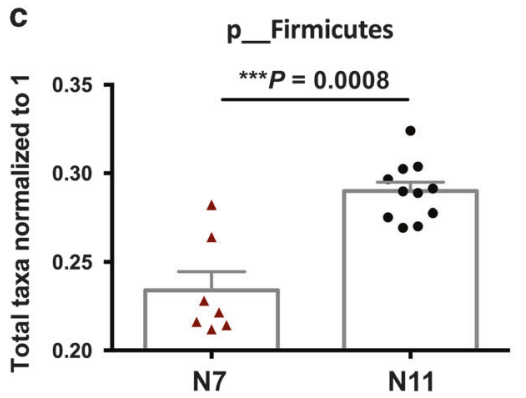

d

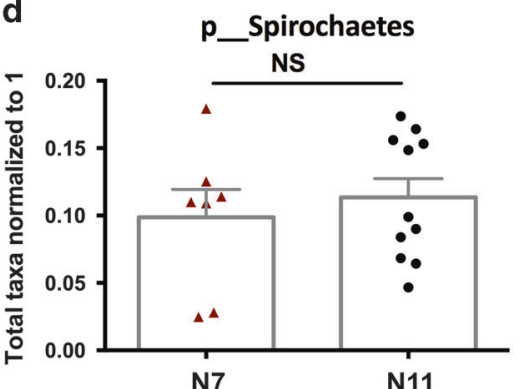

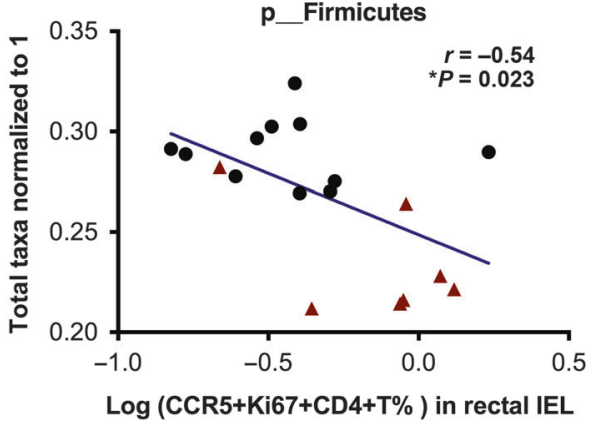

p__Proteobacteria

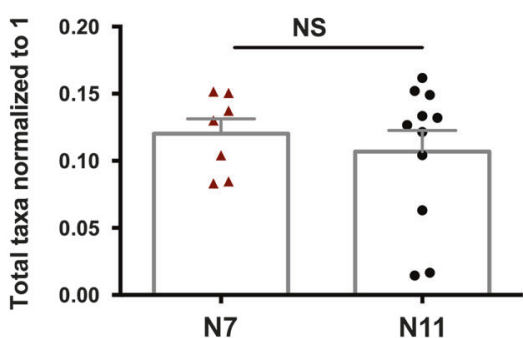

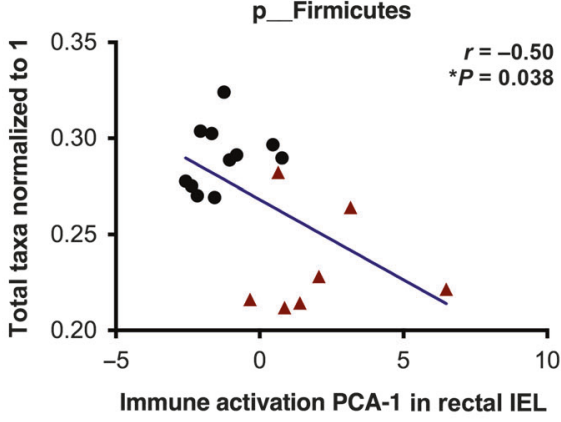

p_Bacteroidetes

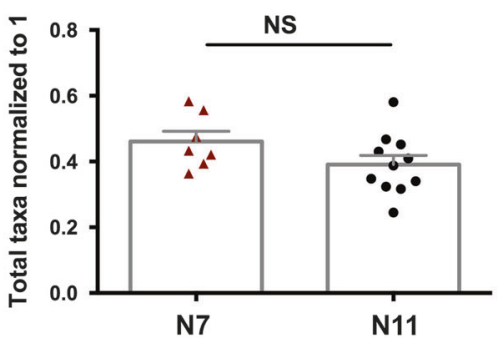

Fig. 3 The analysis of relative abundance of the fecal microbiome at phylum level revealed that Firmicutes were higher in the more viralresistant cohort, and correlated with viral acquisition. a Gut taxa composition from all 18 animals is shown. b Gut taxa composition from each animal is shown. c The relative abundance of Firmicutes was compared between the two cohorts using a Mann-Whitney test. Correlation between relative abundance of Firmicutes and viral target cells and immune activation PCA-1 were performed using Spearman's tests. $\mathbf{d}$ The relative abundance of Spirochaetes, Proteobacteria, and Bacteroidtes were compared between the two cohorts using Mann-Whitney tests. Mean \pm SEM are shown

generated PCA plot revealed that the N7 and the N11 groups had distinct immune activation status (Fig. 2a). Highlighting a common signature of each cohort, the parameters listed in Fig. 2b accounted for most of the difference in the colorectal mucosa. The more resistant group of $\mathrm{N} 11$ had higher expression of CD38 in both CD4 and CD8 T cells, as well as higher HLA-DR expression in $\mathrm{CD} 14^{+}$monocytes, while the more susceptible cohort N7 had higher expression of CCR5 in all three cell types (CD4, CD8 T cells and $\mathrm{CD} 14^{+}$monocytes), and higher Ki67 expression in CD4 T cells. This suggested that the immune signature of the two cohorts primarily involved the expression of CCR5, Ki67, CD38, and HLADR. Overall, the two cohorts of macaques had different expression levels of immune activation markers on T cells and myeloid cells in the rectal mucosa.

We then exported the PCA-1 of the immune activation markers, and compared them in the two naive groups. As predicted, the N7 and N11 groups had significantly different PCA-1, which also showed a significant association with number of exposures needed for infection (Fig. 2c, d).

To summarize, SHIV viral susceptibility was associated with two markers: (1) the prevalence of activated rectal viral target cells, and
(2) the PCA-1 of multiple immune activation markers in the rectal mucosa. Both could be used as surrogate markers to monitor the immune activation status.

Distinct bacterial taxa composition of fecal microbiome in the two naive cohorts

Because the two cohorts of naive groups came from two different sources, we hypothesized that the difference in immune activation in the Gl tract might be due to distinct gut microbiota. To test this hypothesis, we examined the gut microbiome by $16 \mathrm{~S}$ rDNA MiSeq. Total bacterial DNA was extracted from the fecal samples collected one-week before the serial challenges. Using the MiSeq protocol, we obtained an average of $108,096 \pm 3121$ operational taxonomic units (OTUs) per sample, which were subjected to subsequent analysis (Supplementary Table 2). We further examined the bacterial compositions at different levels, and investigated whether the gut microbiome was associated with local mucosal immune activation or SHIV susceptibility.

At the phylum level, 17 phyla have been identified. A compositional look at this level revealed that the top four phyla: Bacteroidetes, Firmicutes, Proteobacteria, and Spirochaetes 
a
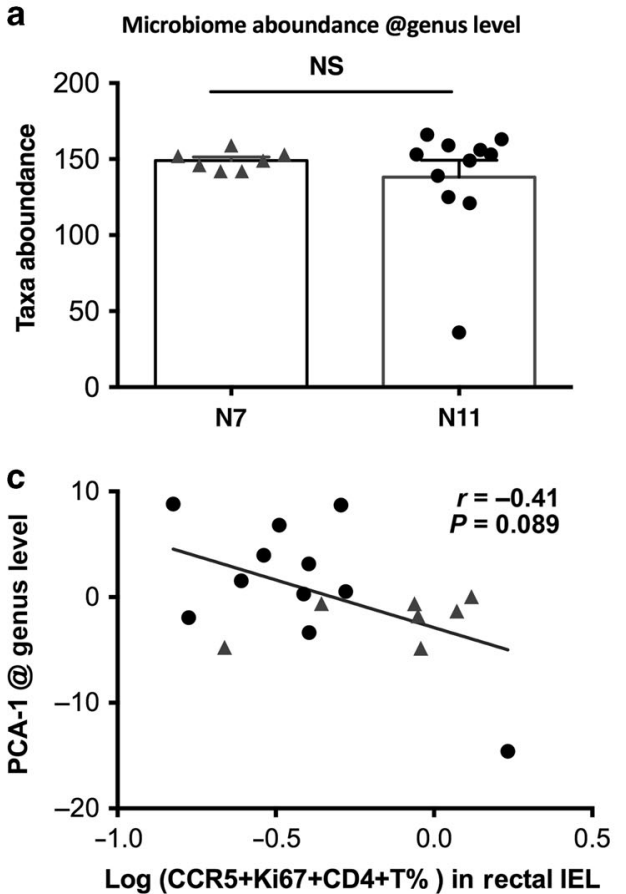
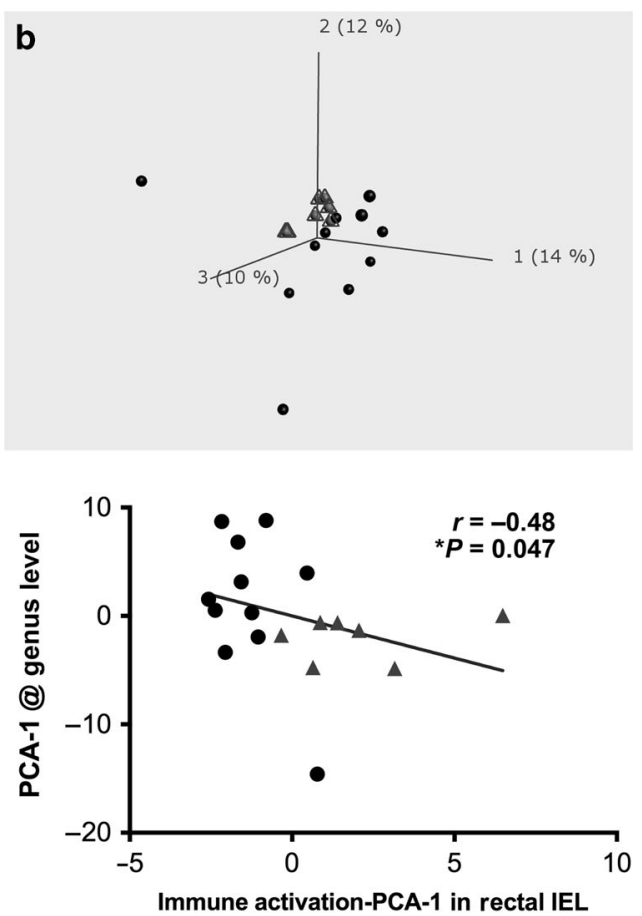

Fig. 4 Principal component analysis (PCA) components at genus level correlated with rectal viral target cells and immune activation PCA-1 (in rectal IEL). a The number of total taxa identified in each animal of the two cohorts were compared using Mann-Whitney test. Mean \pm SEM are shown. b PCA plot of gut microbiome at genus level is shown. Red triangles denote the N7 group, and black dots denote the N11 group. c Correlations between PCA component 1 of gut microbiome at genus level versus viral target cells or immune activation PCA-1 were performed using Spearman's test

represented more than $90 \%$ of the total bacteria (Fig. 3a, b). Among these four phyla, Firmicutes were significantly higher in the N11 group (the resistant cohort) than in the N7 group (Fig. 3c). Furthermore, Firmicutes correlated inversely with a low frequency of $\mathrm{Ki} 67^{+} \mathrm{CCR} 5^{+} \mathrm{CD} 4^{+} \mathrm{T}$ cells (SHIV target cells) in the rectal mucosa and showed a correlation with mucosal immune activation PCA-1 (Fig. 3c), suggesting that Firmicutes might influence the frequencies of viral target cells and immune activation markers in the gut mucosa. However, Firmicutes did not correlate with numbers of exposures to SHIV virus, suggesting they unlikely affected the SHIV viral transmission directly (Supplementary Figure 4). The other top three phyla: Bacteroidetes, Proteobacteria, and Spirochaetes did not differ between the two cohorts (Fig. 3d).

Gut microbiota composition at the genus level showed that the bacterial PCA correlated with viral target cells/immune activation in the rectal mucosa

We then zoomed in on the bacterial compositions at the genus level, in which over 200 taxa were identified. We did not find significantly different taxa abundance between the two cohorts of macaques (Fig. 4a). However, the PCA plot of the gut bacteria showed different microbial compositions of the two cohorts, even after more than 5 months of co-housing (Fig. 4b). Exporting the bacterial PCAs, we found that PCA-1, representing $14 \%$ of total taxa, showed a correlation or a trend of correlation with mucosal immune activation PCA 1 , or $\mathrm{Ki} 67^{+} \mathrm{CCR}^{+} \mathrm{CD} 4^{+} \mathrm{T}$ SHIV target cells (Fig. 4c). However, bacterial PCA-1 did not directly correlate with the number of viral exposures.

Among the taxa at the genus level, Anaerovibrio, Bacteroides, Bulleidia, Ruminococcus, Mogibacterium, and Symbiobacterium were significantly higher in the N11 group, while Anaeroplasma, Burkholderia, syntrophobacter were significantly higher in the N7 group (Fig. 5). We also tried to identify the specific bacteria that might be associated with the viral infection. Mogibacterium,
Hydrogenoanaerobacterium, and Orientia showed corrections with viral acquisition directly (supplementary Figure 5).

The different sources of the two cohorts of animals might account for the different gut microbiome they had. However, we noticed that the average age of $\mathrm{N} 11$ cohort was relatively younger than that of the N7 cohort group (Supplementary Table 1). In the N7 group, three animals had ages similar to those of the N11 group, while the other four were older. Since a previous study has found that old age is associated with microbial dysbiosis and systemic inflammation, ${ }^{32}$ to determine whether age played any roles in determining immune activation and the composition of the gut microbiome, we have included another 21 animals, which were obtained from the same source (Morgan Island) and which arrived in the facility in the same shipment at the same time as the N7 group. As the macaques were all relatively young adults (aging from about 3-5.5 years), not elderly, we did not find any correlations between age and viral target cells in the rectal mucosa or PBMC (Supplementary Figure $6 a-b$ ). Moreover, fecal samples from these 21 animals and the N7 groups were collected and sequenced for microbiome when they first arrived in the facility before any treatment or challenge. We did not observe any associations between age and the composition of gut microbiome either (Supplementary Figure $6 \mathrm{c}-\mathrm{e}$ ). All animals in the N11 cohort were females, whereas there were both males $(N=5)$ and females $(N=2)$ in the N7 cohort. To test whether gender was a major factor in the observed differences in gut bacteria, we analyzed the data and did not find any gender-specific differences in gut bacteria: the two values of the females lay within the range of the five values of the males for PCA- 1 and the Firmicute frequencies ( $P$ $=0.86$ for each).

The ratio Bacteriodes /Prevotella inversely correlated with gut mucosal immune activation

Consistent with a previous report that Prevotella but not Bacteroides is the more common genus in the gut of macaques, ${ }^{33}$ 

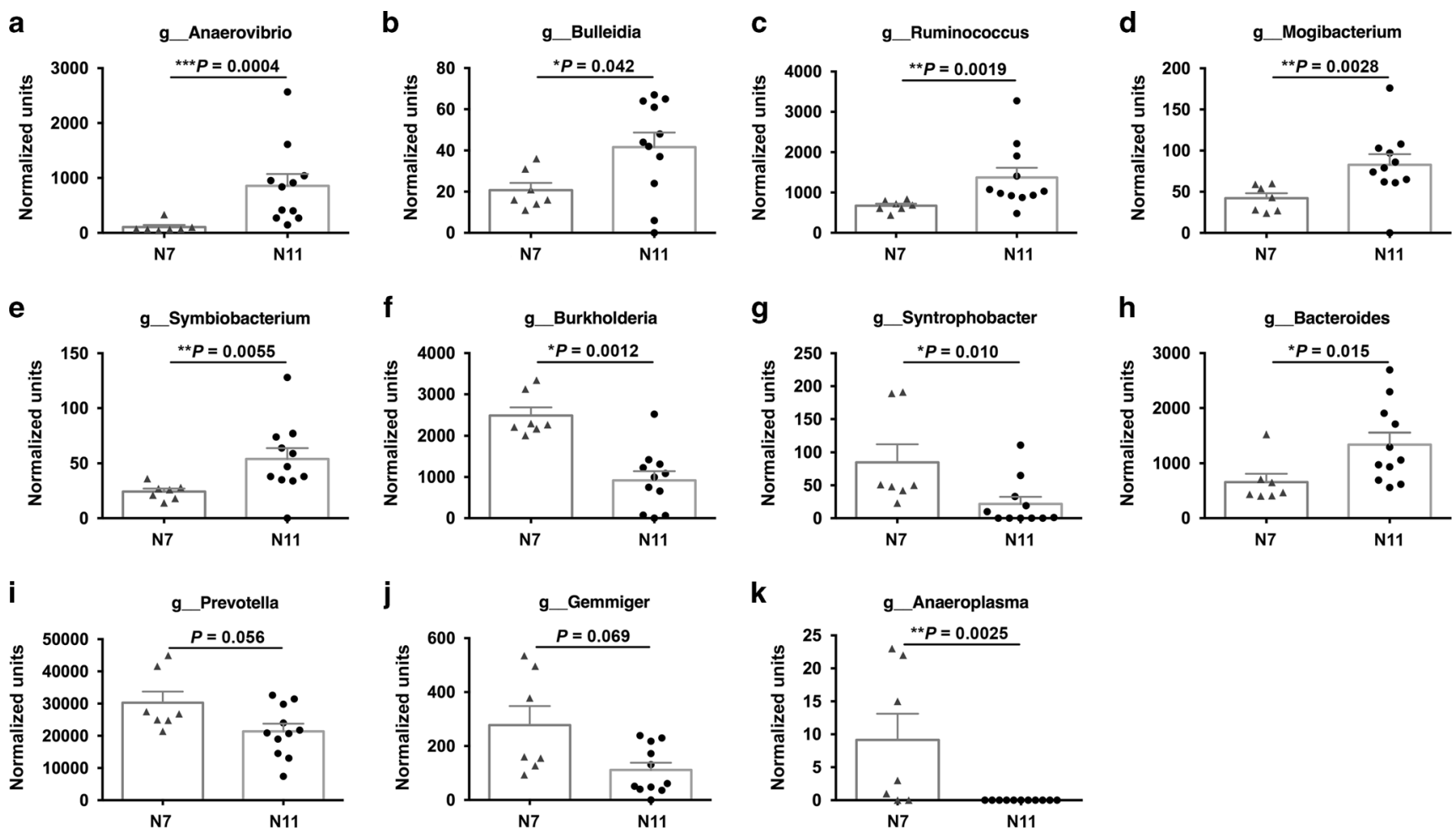

Fig. 5 Relative abundance of the fecal microbiome at genus level was compared in the two naive control groups. Gut bacterial composition at the genus level. a-d Firmicutes, e-g Proteobacteria, $\mathbf{h}$ Bacteroidetes, $\mathbf{i}-\mathbf{k}$ others. Mean \pm SEM are shown, and statistical analyses were performed using Mann-Whitney tests
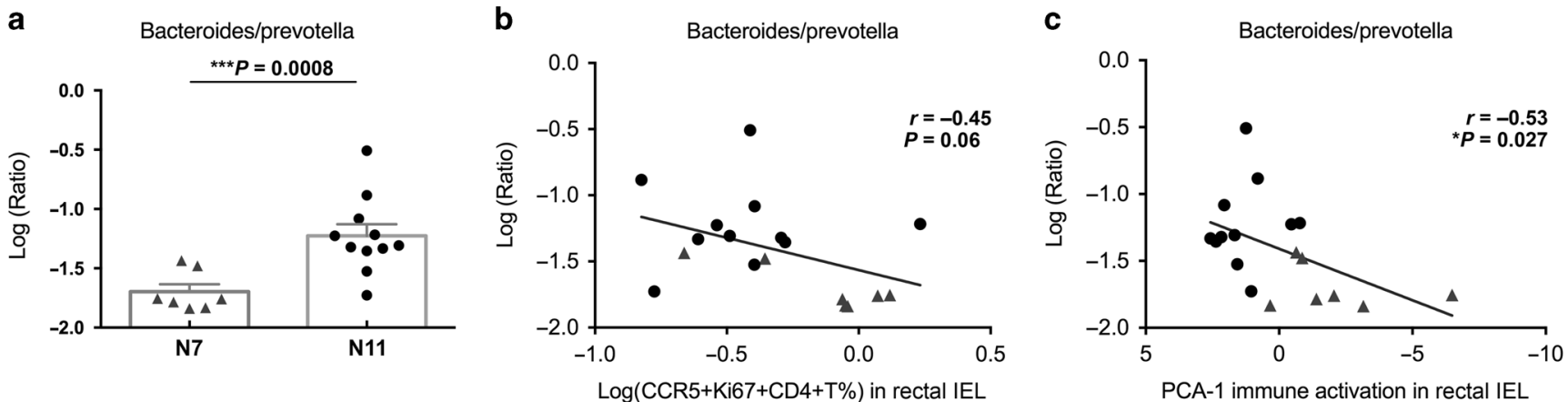

Fig. 6 The ratio of bacteroides/prevotella was higher in the more susceptible cohort and showed corrections with immune activation at genus level. a A Mann-Whitney test was used to compare the ratio of bacteroides/prevotella between the two cohorts. $\mathbf{b}$, $\mathbf{c}$ Spearman's tests were used to determine the correlations between the ratio of bacteroides/prevotella and viral target cells (b) or immune activation (PCA-1) (c) in the rectal IEL

we found that six out of seven macaques from N7 group hardly had any Bacteriodes in the gut (less than 1000 units), whereas more than half of the N11 group had more than 1000 units of Bacteriodes (Fig. 5). Since the ratio of bacteroides to prevotella has been previously shown lower in HIV-1 infected patients, ${ }^{20}$ we compared this ratio in the two cohorts. Notably, the two cohorts had different abundance of Bacteriodes, Prevotella, and the the ratio of bacteroides to prevotella was significantly lower in the more susceptible cohort, as in the HIV-infected patients ${ }^{20}$ (Fig. 6a). Though the ratio of bacteroides to prevotella did not directly correlate with number of viral challenges needed for the animals to get infected, it did show a trend of inverse correlation with viral target cells and did show a significant association with mucosal immune activation PCA-1 (Fig. 6b, c).

We further identified the prevotella and bacteroides species that were different between the two cohorts. Among the ten species that had more than $1 \%$ of the total genus prevotella, $P$. copri ranked first and comprised $65 \%$ of the genus prevotella (Fig. 7a). However, only $P$. histicola, $P$. brevis, $P$. Oris, and $P$. albensis showed significant differences between the N7 and N11 groups (Fig. 7b). Nevertheless, none of them correlated with $\mathrm{Ki} 67^{+} \mathrm{CCR} 5^{+} \mathrm{CD} 4{ }^{+} \mathrm{T}$ cells in the rectal mucosa or mucosal immune activation PCA-1. In the genus bacteroides, the top four species: $B$. intestinalis, B. xylanolyticus, B. plebeius, and B. acidifaciens comprised more than $80 \%$ of the total genus (Fig. 7c). B. intestinalis, and $B$. plebeius showed significant differences between the N7 and N11 groups (Fig. 7d). B. intestinalis was associated with both $\mathrm{Ki} 67^{+} \mathrm{CCR}^{+} \mathrm{CD}^{+} \mathrm{T}$ cells in rectal mucosa and the mucosal immune activation PCA-1 (Fig. 7e). We then asked whether mucosal viral target cells or immune activation PCA-1 correlated with the ratios of $B$. intestinalis to different species of prevotella. The ratios with all four species demonstrated associations with viral target cells or immune activation PCA-1 in the rectal mucosa (Fig. 7f). Overall, at the species level, B. intestinalis not only differed 

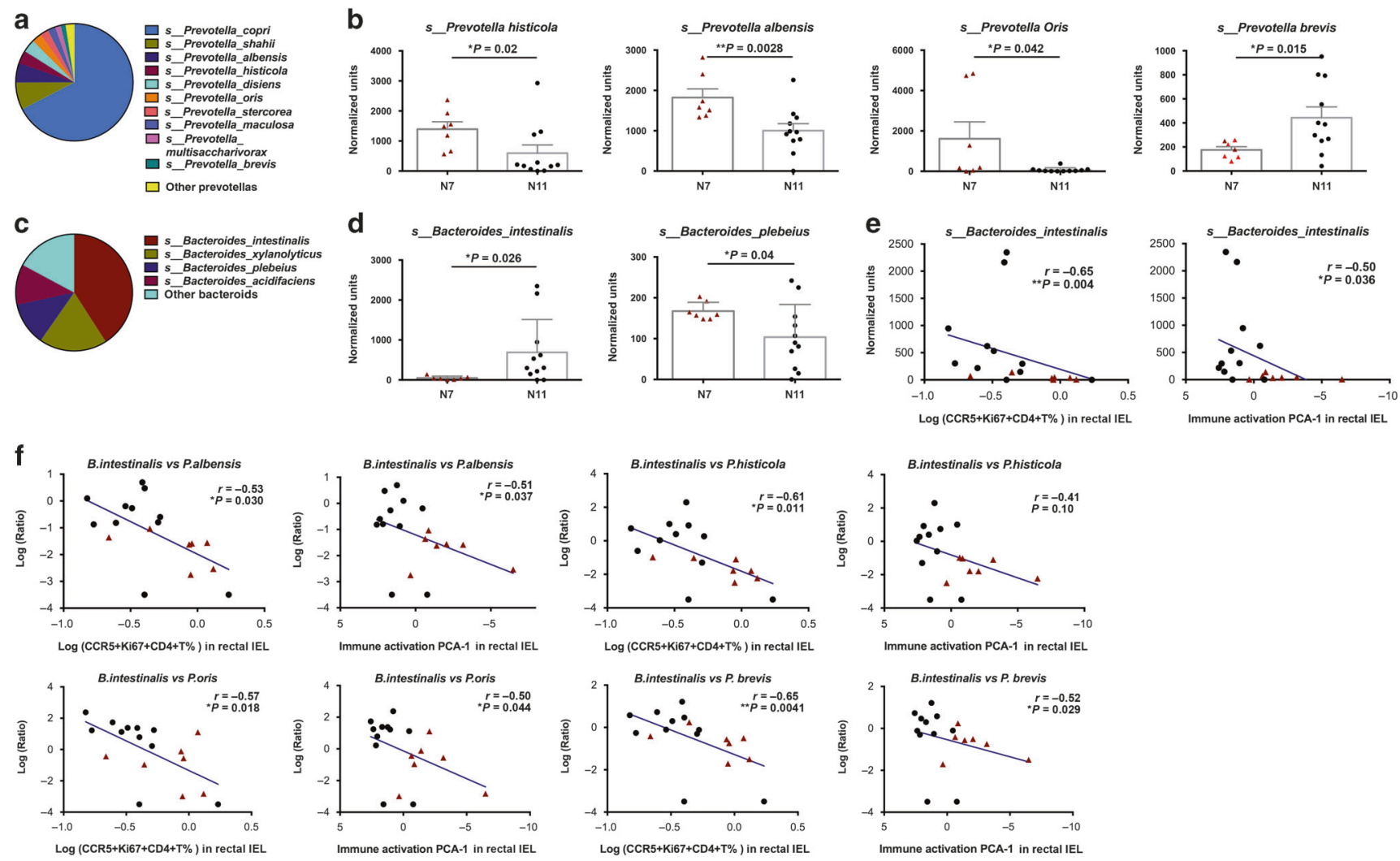

Fig. 7 The specific species of bacteroides and prevotella that correlated with viral target cells or immune activation PCA-1 were identified. a Gut bacterial composition of prevotella at the species level. b The four species of prevotella that showed difference in the two cohorts. $\mathbf{c}$ Gut bacterial composition of bacteroides at the species level. $\mathbf{d}$ The two species of bacteroides that showed differences in the two cohorts. e The species of bacteroides that correlated with viral target cells or immune activation PCA-1 were identified using Spearman's tests. $\mathbf{f}$ Spearman's tests were used to evaluated whether the ratio of specific species of bacteroides/prevotella correlated with the viral target cells or immune activation PCA -1 in the rectal IEL. Mean \pm SEM are shown, and the comparisons of bacterial species between the two cohorts were analyzed using Mann-Whitney tests

in two naive cohorts, but also was associated with viral target cells and mucosal immune activation PCA-1. Future studies should address whether $B$. intestinalis and its metabolites modulate the viral target cells and/or CCR5 expression on T and myeloid cells.

Fecal samples from N7 and N11 groups induced different levels of immune activation markers on $\mathrm{CD}^{+} \mathrm{T}$ cells of naive PBMCs The correlations between immune activation and gut microbiome could not prove that the microbiome differences were causing the immunological differences. To investigate the potential impact of gut microbiome on immune activation markers, we treated naive PBMCs with supernatant of heat-killed fecal samples from each animal of the N7 and N11 groups, and compared the expression levels of immune activation markers on T cells, especially the viral target $\mathrm{CD} 4^{+} \mathrm{T}$ cells $\left(\mathrm{CCR} 5^{+} \mathrm{Ki} 67^{+}\right)$. After co-culture, the frequencies of $\mathrm{Ki} 67^{+}, \mathrm{CD} 8^{+}, \mathrm{CD} 69^{+}$, and $\mathrm{CCR}^{+} \mathrm{Ki}^{+} 7^{+}$were significantly lower on $\mathrm{CD}^{+}{ }^{+} \mathrm{T}$ cells stimulated with fecal samples from N11 group than those from N7 group, while the expression level of CCR5 and HLA-DR alone on $\mathrm{CD}^{+}{ }^{+}$T cells were similar (Fig. 8). Importantly, the frequency of $\mathrm{CD}^{+}{ }^{+} \mathrm{T}$ cells was not differentially affected by the microbiota from the two groups of macaques, indicating that the lower levels of activation by the N11 group fecal samples was not due to gross toxicity to $\mathrm{CD}^{+}{ }^{+} \mathrm{T}$ cells (Fig. $8 \mathrm{f}$ ). Most of the immune activation markers on $\mathrm{CD}^{+} \mathrm{T}$ cells did not significantly differ between N7 and N11 groups after stimulated with fecal samples from each group (Fig. 8, supplementary Figure 7). However, the $\mathrm{CD}^{+} \mathrm{T}$ cell frequency among the $\mathrm{CD}^{+} 5^{+}$live cell gating was significantly decreased after co-culture with fecal samples from N11 group, compared to the N7 group. Overall, exposure of naive
PBMC CD4 ${ }^{+}$T cells to fecal bacteria from N7 and N11 cohorts resulted in significantly different expression levels of immune activation markers, consistent with the expression patterns observed in vivo. This result supported the interpretation that the gut bacterial composition might contribute to the immune phenotypes and/or infection outcomes of this study and could explain the correlations observed.

\section{DISCUSSION}

Here we have found that rhesus macaques from different sources have different microbiota, which correlate with levels of immune activation in the rectal mucosa, which in turn affects the susceptibility of the macaques to rectal transmission of SIV. We observed that the more susceptible N7 group had significantly more activated $\mathrm{CD} 4^{+} \mathrm{CCR} 5^{+} \mathrm{Ki} 67^{+} \mathrm{T}$ cells in the rectal mucosa than the more resistant group of $\mathrm{N} 11$. The groups also differed significantly by PCA of other activation markers. Moreover, these animals demonstrated different HIV/AIDS susceptibility in a lowdose SHIV challenge model. The prevalence of these activated rectal CD4 T cells and the PCA-1 of other immune activation markers in the rectal mucosa inversely correlated with the number of challenges required to infect. After sequencing 16s rRNA, the preliminary PCA of the gut taxa showed that these two cohorts had different microbial compositions, which were associated with gut immune activation, providing more activated viral target cells for SHIV infection. Significantly lower ratios of Bacteroides to Prevotella, and significantly lower levels of Firmicutes were found in the susceptible N7 cohort. These parameters also inversely 
a

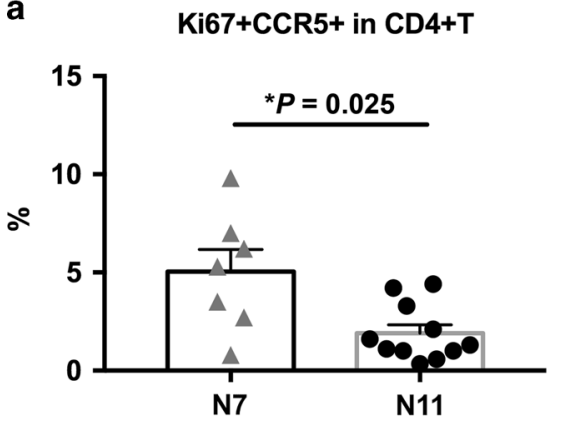

d

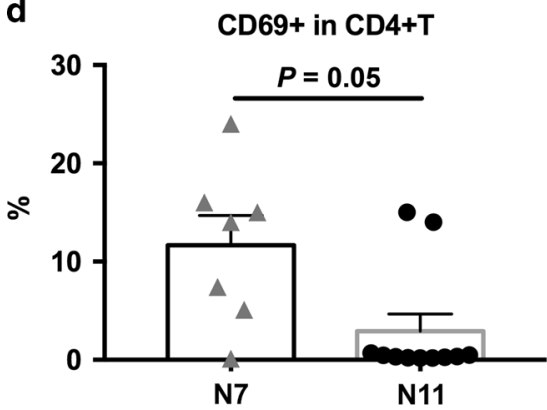

g

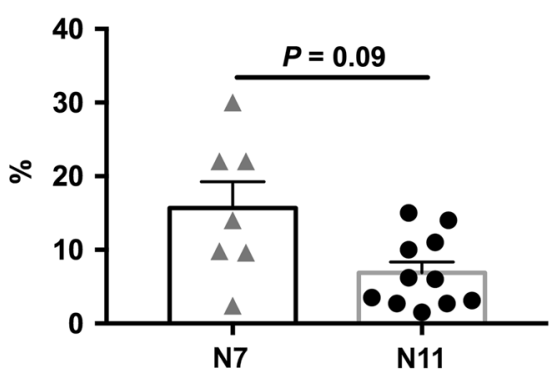

j

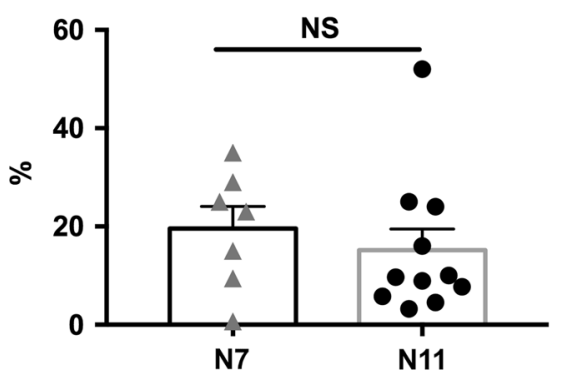

b

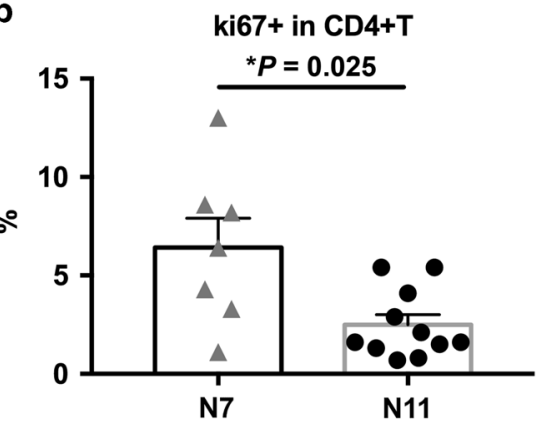

e

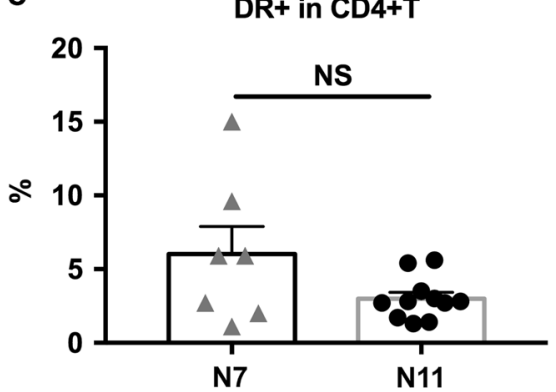

h

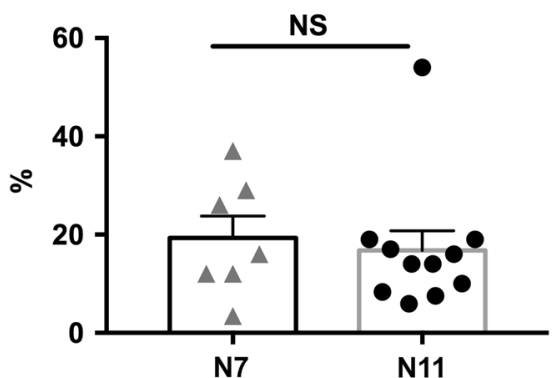

k

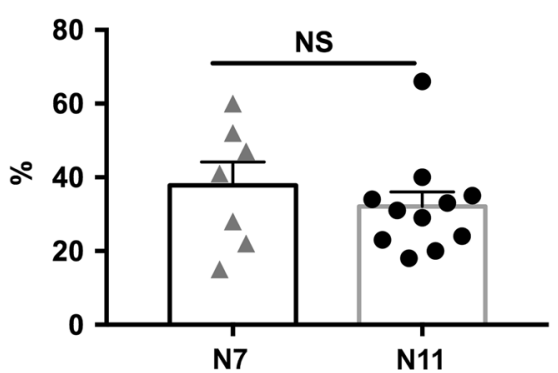

C CD38+ in CD4+T

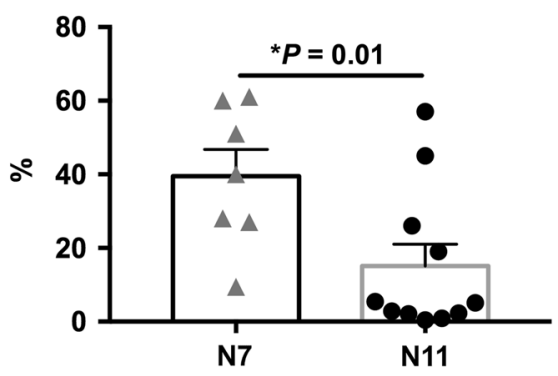

f

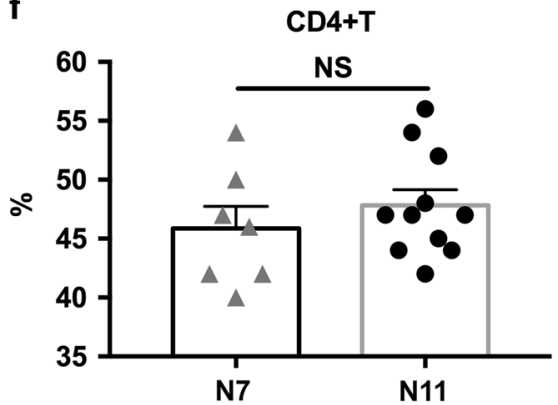

i

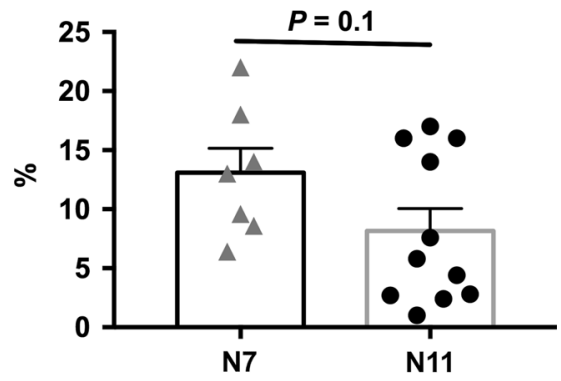

I

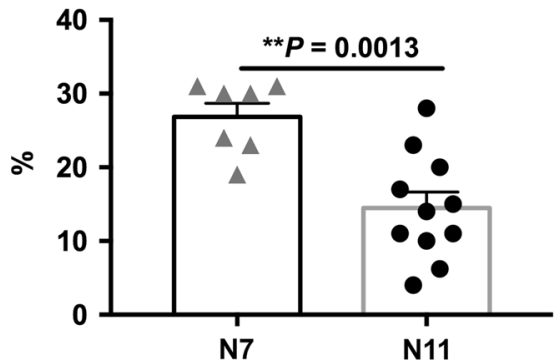

Fig. 8 Ex vivo co-culture of heat-killed gut microbiota from N7 and N11 groups with naive PBMCs resulted in significantly different levels of immune activation markers on T cells. The supernatant of heat-killed gut microbiota from each animal of the N7 and N11 groups were cocultured with naive PBMC for $48 \mathrm{~h}$ as described in Materials and methods section. Cells were harvested and the frequencies of immune activated markers $\mathrm{Ki}^{+}{ }^{+}, \mathrm{CCR}^{+}, \mathrm{Ki}^{+} 7^{+}, \mathrm{CD}^{+} 8^{+}, \mathrm{CD}^{+} 9^{+}$, and $\mathrm{HLA}^{-D R}{ }^{+}$on $\mathrm{CD}^{+}(\mathbf{a}-\mathbf{f})$ and $\mathrm{CD}^{+}$(g-I) $\mathrm{T}$ cells were evaluated using flow cytometry. CD69 on $\mathrm{CD} 4^{+} \mathrm{T}$ cells, which peaks earlier, showed ${ }^{*} P=0.02$ for N7 $>\mathrm{N} 11$ at $18 \mathrm{~h}$ after treatment. Means \pm SEM are shown, and the comparisons between N7 and N11 groups were analyzed using Mann-Whitney tests

correlated with high levels of immune activation markers as well as viral target $\mathrm{CD} 4^{+} \mathrm{T}$ cells in rectal mucosa. In field epidemiology studies, genital microbiome, from either female ${ }^{34,35}$ or male $\mathrm{e}^{36}$ genital tracts, has been correlated with HIV acquisition risk. Furthermore, vaginal bacteria have been shown to affect the microbicide efficacy against HIV infection via bacterial metabolism of the microbicide, leading to tenofovir depletion. ${ }^{37}$ Whether gut microbiome will do the same in the case of intrarectal infection is an open question. Our study demonstrated for the first time that macaques with distinct gut microbiota and immune activation markers had different endogenous mucosal SHIV infection risks when challenged intrarectally with identical doses of the same SHIV.

Chronic immune activation in HIV/AIDS is associated with high viral loads and CD4 T cell depletion, and can determine the progression of AIDS disease. ${ }^{38}$ Mucosal immune activation status, 
especially the viral target cells in the colorectal mucosa, plays an even more important role in determining the outcomes of HIV-1 infection than the corresponding peripheral parameters do. This prompted us to investigate the immune activation status in the colorectal mucosa in the two naive cohorts. Indeed, the two cohorts had different levels of mucosal immune activation markers, which correlated with viral susceptibility. To identify the cause of these differences between the two cohorts of naive animals, we proposed that the gut microbiome might be responsible. In HIV infection, gut microbial translocation has been implicated as a major driving force to contribute to chronic immune activation. ${ }^{39}$ The bacteria or the bacterial products moving from the gut lumen to LP and systemic circulation interrupted the delicate balance between immunogenic and tolerogenic intestinal immune responses. Furthermore, plasma bacterial lipopolysaccharide (LPS) levels correlated with measures of innate and adaptive immune activation in chronically HIVinfected individuals and in SIV-infected rhesus macaques. ${ }^{40}$ In naive animals, we do not expect to observe as much gut microbial translocation as in the HIV/SIV infected ones. Nevertheless, the gut microbiome and its metabolites can interact with the host immune cells in the gut mucosa via a diversity of receptors, including Toll-like receptors (TLRs), NOD-like receptors (NLRs), and C-type lectin receptors, such as dectin 1, dectin 2, and macrophage-inducible C-type lectin. ${ }^{41-43}$ Specific gut microbiota are able to transduce specific signals in the host immune cells, and affect the immune activation of these cells. After exposure to Prevotella stercorea, naive LP CD4 ${ }^{+} \mathrm{T}$ cells upregulated Ki67, p38 MAPK, and HIV viral DNA replication host co-factors, which led to increased HIV viral susceptibility in vitro. ${ }^{44}$ In the current in vivo study, we found that two cohorts of naive animals with different gut microbiomes and immune activation markers, that correlated with each other, had different infection rates for SHIV challenge. However, correlation alone can never prove causation. To find possible further evidence for a causative effect, we did an ex vivo co-culture experiment to evaluate the impact of the gut microbiome community and its metabolites on $\mathrm{T}$ cell immune activation. We found that supernatant of heat-killed fecal samples from the more susceptible cohort N7 group induced overall higher expression levels of immune activation markers (Ki67, CD38, CD69) on $\mathrm{CD}^{+}{ }^{+} \mathrm{T}$ cells from naive PBMCs than that of the N11 group. Furthermore, the frequency of viral target cells, $\mathrm{Ki}^{+} 7^{+} \mathrm{CCR}^{+}$ $\mathrm{CD} 4^{+} \mathrm{T}$ cells, was significantly higher in PBMCs treated with fecal bacterial samples from the N7 group than those from the N11 group. Though we did not dissect which bacteria were responsible for the changes, these data suggested that the gut microbiome as a whole might modulate the viral susceptibility via altering the expression levels of immune activation markers on T cells as well as the prevalence of viral target cells. Indeed, Prevotella may be playing a role here as well because the ratio of Bacterioides to Prevotella species correlated with immune activation. Analysis of specific bacteria will be the topic of future research.

In this study, we did not find a significant difference in Treg cells in the rectal mucosa of the two cohorts. Instead, the two cohorts had different expression levels of immune activation markers, such as CCR5, ki67, and CD38 on different cell subsets, particularly $\mathrm{T}$ and myeloid cells, suggesting the possible alteration of these markers on different cell types by gut microbiota or their metabolites. Activated or proliferating $\mathrm{CCR}^{+}{ }^{+} \mathrm{CD} 4^{+} \mathrm{T}$ cells in the colorectal tissues have been shown to inversely correlate with SIV viral eclipse time ${ }^{30}$ and to predict increased SIV viral acquisition in the SIV-vaccinated rhesus macaques. ${ }^{29}$ However, in vaccine studies, the vaccine induced antigen-specific immune responses might be a confounder in interpreting the results. In this study, using two groups of naive macaques, we demonstrated that the immune activation status of the colorectal mucosa was associated with SHIV viral acquisition. Since all the animals were naive, and never exposed previously to SHIV viral antigens, viral-specific immune responses can be ruled out as a factor to determine the challenge outcome.

The finding that naive animals from different sources demonstrated significantly different HIV/SIV viral susceptibility poses a big problem in HIV/AIDS research. Though we cannot determine the absolute role of each of the examined parameters, the gut microbiome, viral target cells, and mucosal immune activation markers in the rectal tissue should all be measured. One direct implication of this study is the need to set up new criteria for choosing macaques for future HIV/SIV vaccine efficacy or challenge studies. To be matched, the macaques need to have not only similar gender, age and MHC haplotypes, but also similar gut microbiome and prevalence of viral target cells/immune activation in the mucosa. Another important implication is that future studies are needed to take the gut microbiota prevalent in different human populations into account in assessing HIV-1 susceptibility. Stool specimens should be collected for all HIV transmission studies and clinical trials to evaluate this variable. Probiotics may be able to be designed to reduce transmission risk. To the extent that this parameter may be hard to alter in vaccinees, it should be taken into account when comparing vaccine efficacy in different populations with different diets and microbial exposures.

Overall, this study provides novel insights into microbiome-host interactions, and paves the way for potential strategies to reduce susceptibility to HIV-1 transmission or improve vaccine efficacy through microbial manipulation.

\section{MATERIALS AND METHODS}

Animals

Two cohorts of adult Indian rhesus macaques (Macaca mulatta) were used with the approval of Institutional Animal Care and Use Committees. The macaques were housed at the $\mathrm{NCl}$ Animal Facility, Bethesda, MD (Protocols No. VB011 approved by the $\mathrm{NCl}$ IACUC). The facility is accredited by the Association for Assessment and Accreditation of Laboratory Animal Care (AAALAC) International. The recommendations of the Standards and Guide for the Care and Use of Laboratory Animals of the United States were closely followed. One cohort of seven naive macaques (N7 group) was obtained from Morgan Island, while the other 11 naive macaques were obtained from Alpha Genesis Inc. The detail information of the animals is listed in Supplementary Table 1. PBMC and rectal pinch biopsies were collected to measure immune activation. Fecal samples were also collected one week before SHIV challenge.

Viral challenge, and viral load (VL) measurements

The animals were intrarectally exposed to eight serial SHIV $\mathrm{SF}_{162 \mathrm{P} 4}$ viral challenges (1:35 dilution) at one-week intervals until they were infected. The SHIV $\mathrm{SF}_{162 \mathrm{P} 4}$ viral stock was kindly provided by Nancy Miller of the National Institute of Allergy and Infectious Diseases (NIAID). After the viral challenges, SHIV RNA levels were monitored by Advanced BioScience Laboratories, Inc. The cut-off threshold for viral RNA detection was 50 copies $/ \mathrm{ml}$.

\section{Flow cytometry analysis of single-cell suspensions from rectal} pinches

Single-cell suspensions from rectal pinches were prepared as previously described. ${ }^{30}$ Rectal IEL and LP were collected and subjected to flow cytometry analysis. The single-cell suspensions were first incubated with Fc Receptor blocking reagent (Miltenyi Biotec), followed by staining with viability dye (Invitrogen). The antibody mixtures were then incubated as previously described. ${ }^{27}$ For immune activation, the following antibodies were used: CD45PerCP, CD3-PE-Cy7, CD4-BV605, CD8-APC-Cy7, CD14-V450, Ki67APC, HLA-DR PE-Cy5, and CCR5-PE (BD Pharmingen); CD69-Alexa Fluor 700 (Biolegend); and CD38-FITC (STEMCELL Technologies). 
For detection of Treg and MDSCs, the following antibody mixture were used: CD45-PerCP/Cy5.5, CD3-PE-Cy7, CD4-BV605, CD8BV785, lin 1-FITC (BD Pharmingen), FOXP3-APC (eBioscience), HLA-DR-APC-Cy7, CD11b-PE-Cy5, CD14-BV711, CD8-BV785, CD25BV421, CD15-Alexa700 (Biolegend), CD33-PE (Milteny). An LSRII flow cytometer was used for data acquisition. FlowJo software (Tree Star Inc.) was used for data analyses.

\section{Sample processing and sequencing for gut microbiome}

Fecal samples were collected one-week before the serial SHIV viral challenges. DNA was extracted from $\sim 200 \mathrm{mg}$ of stool using the Qiagen DNA Stool Mini Kit. DNA amplification was performed using Eppendorf liquid handling robots. The V4 region of the $16 \mathrm{~S}$ rDNA gene (515F-806R) was sequenced for 18 samples; generating paired-end, overlapping reads on the Illumina MiSeq platform. ${ }^{45}$

Chimeric sequences were filtered out of the FASTQ files containing the 16S rRNA gene sequences using the USEARCH (version 8.1.1831) $^{46}$ utility's UCHIME $^{47}$ implementation and the 'gold' database (version microbiomeutil-r20110519). The reads, thus filtered, were then binned into OTUs at 97\% similarity using USEARCH's cluster_otus command. QIIME (1.9.1) ${ }^{48}$ scripts were used to classify and align the obtained OTUs. The assign_taxonomy.py script was used to assign taxonomy using the default RDP method ${ }^{49}$ and GreenGenes database. ${ }^{50}$

\section{PBMC stimulation with fecal samples}

Fecal samples from each animal of the N7 and N11 groups were collected one week before SHIV viral challenges. One gram fecal samples (wet weight) were diluted with $1 \mathrm{ml}$ of PBS and homogenized for $5 \mathrm{~min}$. The homogenized fecal samples were then heat-treated at $56^{\circ} \mathrm{C}$ for $2 \mathrm{~h}$ to kill the bacteria. Following heat-treatment, the fecal samples were subjected to a low-spin centrifugation ( $2000 \mathrm{rpm}, 2 \mathrm{~min}$ ) to remove large particulate material. The supernatant fractions were collected, and stored at single-use aliquots at $-20^{\circ} \mathrm{C}$.

Fresh PBMCs from naive rhesus macaques were suspended in R10 medium to a final concentration of 2 million $/ \mathrm{ml}$ in a 48-well plate, $500 \mu \mathrm{l}$ per well, at $37^{\circ} \mathrm{C}, 5 \% \mathrm{CO}_{2}$. Fifty five microliter of supernatant of fecal sample preparations were added to each well, and cultured for 18 or $48 \mathrm{~h}$. After stimulation, the cells were washed twice with FACS buffer, and then subjected to flow cytometry analysis as described above. The experiments were performed twice for each time point independently. CD69 was best measured at $18 \mathrm{~h}$ but the other markers shown were highest at $48 \mathrm{~h}$.

\section{Statistical analysis and data visualization}

Principal component analyses (PCA) were performed with Qlucore Omics Explorer (Qlucore) or SAS. Spearman's correlations were used to analyze the correlations. Mann-Whitney tests were used to compare the difference between N7 and N11 groups (GraphPad Prism software).

\section{AUTHOR CONTRIBUTIONS}

Y.S., J.A.B. designed research studies, Y.S., A.D., B.F. conducted experiments, Y.S., A.D. acquired the data, Y.S., D.V., A.D., J.A.B., G.T., and V.T. analyzed the data, Y.S. wrote original draft, and Y.S., J.A.B. wrote the manuscript.

\section{ADDITIONAL INFORMATION}

The online version of this article (https://doi.org/10.1038/s41385-018-0029-0) contains supplementary material, which is available to authorized users.

Competing interests: The authors declare no competing interests.

Publisher's note: Springer Nature remains neutral with regard to jurisdictional claims in published maps and institutional affiliations.

\section{REFERENCES}

1. Brown, E. M., Sadarangani, M. \& Finlay, B. B. The role of the immune system in governing host-microbe interactions in the intestine. Nat. Immunol. 14, 660-667 (2013).

2. Hooper, L. V., Littman, D. R. \& Macpherson, A. J. Interactions between the microbiota and the immune system. Science 336, 1268-1273 (2012).

3. Strober, W. Impact of the gut microbiome on mucosal inflammation. Trends Immunol. 34, 423-430 (2013).

4. Hsiao, E. Y. et al. Microbiota modulate behavioral and physiological abnormalities associated with neurodevelopmental disorders. Cell 155, 1451-1463 (2013).

5. Hooper, L. V. Bacterial contributions to mammalian gut development. Trends Microbiol. 12, 129-134 (2004).

6. Kabat, A. M., Srinivasan, N. \& Maloy, K. J. Modulation of immune development and function by intestinal microbiota. Trends Immunol. 35, 507-517 (2014).

7. Hill, D. A. \& Artis, D. Intestinal bacteria and the regulation of immune cell homeostasis. Annu. Rev. Immunol. 28, 623-667 (2010).

8. Atarashi, K. et al. Induction of colonic regulatory T cells by indigenous Clostridium species. Science 331, 337-341 (2011).

9. Atarashi, $\mathrm{K}$. et al. ATP drives lamina propria $\mathrm{T}(\mathrm{H}) 17$ cell differentiation. Nature $\mathbf{4 5 5}$, 808-812 (2008).

10. Shaw, M. H., Kamada, N., Kim, Y. G. \& Nunez, G. Microbiota-induced IL-1 beta, but not IL-6, is critical for the development of steady-state TH17 cells in the intestine. J. Exp. Med. 209, 251-258 (2012).

11. Arpaia, N. et al. Metabolites produced by commensal bacteria promote peripheral regulatory T-cell generation. Nature 504, 451-455 (2013).

12. Furusawa, Y. et al. Commensal microbe-derived butyrate induces the differentiation of colonic regulatory T cells. Nature 504, 446-450 (2013).

13. Smith, P. M. et al. The microbial metabolites, short-chain fatty acids, regulate colonic Treg cell homeostasis. Science 341, 569-573 (2013).

14. Round, J. L. \& Mazmanian, S. K. Inducible Foxp3+regulatory T-cell development by a commensal bacterium of the intestinal microbiota. Proc. Natl Acad. Sci. USA 107, 12204-12209 (2010).

15. Round, J. L. et al. The Toll-like receptor 2 pathway establishes colonization by a commensal of the human microbiota. Science 332, 974-977 (2011).

16. Rahimpour, R. et al. Bacterial superantigens induce down-modulation of CC chemokine responsiveness in human monocytes via an alternative chemokine ligand-independent mechanism. J. Immunol. 162, 2299-2307 (1999).

17. Vesterbacka, J. et al. Richer gut microbiota with distinct metabolic profile in HIV infected Elite Controllers. Sci. Rep. 7, 6269 (2017).

18. Vujkovic-Cvijin, I. et al. Dysbiosis of the gut microbiota is associated with HIV disease progression and tryptophan catabolism. Sci. Transl. Med 5, 193 ra191 (2013).

19. Ellis, C. L. et al. Molecular characterization of stool microbiota in HIV-infected subjects by panbacterial and order-level $16 \mathrm{~S}$ ribosomal DNA (rDNA) quantification and correlations with immune activation. J. Acquir. Immune Defic. Syndr. 57, 363-370 (2011).

20. Dillon, S. M. et al. An altered intestinal mucosal microbiome in HIV-1 infection is associated with mucosal and systemic immune activation and endotoxemia. Mucosal Immunol. 7, 983-994 (2014).

21. Glavan, T. W. et al. Gut immune dysfunction through impaired innate pattern recognition receptor expression and gut microbiota dysbiosis in chronic SIV infection. Mucosal Immunol. 9, 677-688 (2016).

22. Handley, S. A. et al. SIV infection-mediated changes in gastrointestinal bacterial microbiome and virome are associated with immunodeficiency and prevented by vaccination. Cell Host Microbe 19, 323-335 (2016).

23. Vazquez-Castellanos, J. F. et al. Altered metabolism of gut microbiota contributes to chronic immune activation in HIV-infected individuals. Mucosal Immunol. 8, 760-772 (2015)

24. McDermott, A. B. et al. Repeated low-dose mucosal simian immunodeficiency virus SIVmac239 challenge results in the same viral and immunological kinetics as high-dose challenge: a model for the evaluation of vaccine efficacy in nonhuman primates. J. Virol. 78, 3140-3144 (2004).

25. Hudgens, M. G. \& Gilbert, P. B. Assessing vaccine effects in repeated low-dose challenge experiments. Biometrics 65, 1223-1232 (2009).

26. Hall, J. A. et al. Commensal DNA limits regulatory $T$ cell conversion and is a natural adjuvant of intestinal immune responses. Immunity 29, 637-649 (2008).

27. Sui, Y. et al. Paradoxical myeloid-derived suppressor cell reduction in the bone marrow of SIV chronically infected macaques. PLoS Pathog. 13, e1006395 (2017).

28. Sui, Y. et al. Vaccine-induced myeloid cell population dampens protective immunity to SIV. J. Clin. Invest. 124, 2538-2549 (2014).

29. Carnathan, D. G. et al. Activated $C D 4+C C R 5+T$ cells in the rectum predict increased SIV acquisition in SIVGag/Tat-vaccinated rhesus macaques. Proc. Natl Acad. Sci. USA 112, 518-523 (2015).

30. Sui, Y. et al. Early SIV dissemination after intrarectal SIVmac251 challenge was associated with proliferating virus-susceptible cells in the colorectum. J. Acquir. Immune Defic. Syndr. 71, 353-358 (2016). 
31. Andersson, A. et al. Molecular signatures in childhood acute leukemia and their correlations to expression patterns in normal hematopoietic subpopulations. Proc. Natl Acad. Sci. USA 102, 19069-19074 (2005).

32. Thevaranjan, $\mathrm{N}$. et al. Age-associated microbial dysbiosis promotes intestinal permeability, systemic inflammation, and macrophage dysfunction. Cell Host Microbe 21, 455-466 e454 (2017)

33. McKenna, P. et al. The macaque gut microbiome in health, lentiviral infection, and chronic enterocolitis. PLoS Pathog. 4, e20 (2008).

34. Gosmann, C. et al. Lactobacillus-deficient cervicovaginal bacterial communities are associated with increased HIV Acquisition in young South African women. Immunity 46, 29-37 (2017).

35. McClelland R. S. et al. Evaluation of the association between the concentrations of key vaginal bacteria and the increased risk of HIV acquisition in African women from five cohorts: a nested case-control study. Lancet Infect Dis 2018. published online, https://doi.org/10.1016/S1473-3099(18)30058-6.

36. Liu C. M. et al. Penile Anaerobic Dysbiosis as a Risk Factor for HIV Infection. MBio 8, e00996-17 (2017).

37. Klatt, N. R. et al. Vaginal bacteria modify HIV tenofovir microbicide efficacy in African women. Science 356, 938-945 (2017).

38. Hunt, P. W. HIV and inflammation: mechanisms and consequences. Curr. HIV/Aids Rep. 9, 139-147 (2012).

39. Marchetti, G., Tincati, C. \& Silvestri, G. Microbial translocation in the pathogenesis of HIV infection and AIDS. Clin. Microbiol. Rev. 26, 2-18 (2013).
40. Brenchley, J. M. et al. Microbial translocation is a cause of systemic immune activation in chronic HIV infection. Nat. Med. 12, 1365-1371 (2006).

41. Brubaker, S. W., Bonham, K. S., Zanoni, I. \& Kagan, J. C. Innate immune pattern recognition: a cell biological perspective. Annu. Rev. Immunol. 33, 257-290 (2015).

42. Kieser, K. J. \& Kagan, J. C. Multi-receptor detection of individual bacterial products by the innate immune system. Nat. Rev. Immunol. 17, 376-390 (2017).

43. Geijtenbeek, T. B. \& Gringhuis, S. I. Signalling through C-type lectin receptors: shaping immune responses. Nat. Rev. Immunol. 9, 465-479 (2009).

44. Yoder, A. C. et al. The transcriptome of HIV-1 infected intestinal CD4+T cells exposed to enteric bacteria. PLoS Pathog. 13, e1006226 (2017).

45. Caporaso, J. G. et al. Global patterns of $16 \mathrm{~S}$ rRNA diversity at a depth of millions of sequences per sample. Proc. Natl Acad. Sci. USA 108, 4516-4522 (2011).

46. Edgar, R. C. Search and clustering orders of magnitude faster than BLAST. Bioinformatics 26, 2460-2461 (2010).

47. Edgar, R. C., Haas, B. J., Clemente, J. C., Quince, C. \& Knight, R. UCHIME improves sensitivity and speed of chimera detection. Bioinformatics 27, 2194-2200 (2011).

48. Caporaso, J. G. et al. QIIME allows analysis of high-throughput community sequencing data. Nat. Methods 7, 335-336 (2010).

49. Wang, Q., Garrity, G. M., Tiedje, J. M. \& Cole, J. R. Naive Bayesian classifier for rapid assignment of rRNA sequences into the new bacterial taxonomy. Appl. Environ. Microbiol. 73, 5261-5267 (2007).

50. DeSantis, T. Z. et al. Greengenes, a chimera-checked $16 \mathrm{~S}$ rRNA gene database and workbench compatible with ARB. Appl. Environ. Microbiol. 72, 5069-5072 (2006). 